\title{
Priming with intranasal lactobacilli prevents Pseudomonas aeruginosa acute pneumonia in mice
}

Marie-Sarah Fangous ${ }^{1,2}$, Philippe Gosset ${ }^{3}$, Nicolas Galakhoff², Stéphanie Gouriou², Charles-Antoine Guilloux², Christopher Payan ${ }^{2,4}$, Sophie Vallet ${ }^{2,4}$, Geneviève Héry-Arnaud ${ }^{2,4}$ and Rozenn Le Berre ${ }^{2,5^{*}}$

\begin{abstract}
Background: Increasing resistance to antibiotics of Pseudomonas aeruginosa leads to therapeutic deadlock and alternative therapies are needed. We aimed to evaluate the effects of Lactobacillus clinical isolates in vivo, through intranasal administration on a murine model of Pseudomonas aeruginosa pneumonia.

Results: We screened in vitro 50 pulmonary clinical isolates of Lactobacillus for their ability to decrease the synthesis of two QS dependent-virulence factors (elastase and pyocyanin) produced by Pseudomonas aeruginosa strain PAO1.

Two blends of three Lactobacillus isolates were then tested in vivo: one with highly effective anti-PAO1 virulence factors properties (blend named L.rff for L. rhamnosus, two L. fermentum strains), and the second with no properties (blend named L.psb, for L. paracasei, L. salivarius and L. brevis). Each blend was administered intranasally to mice 18 h prior to PAO1 pulmonary infection. Animal survival, bacterial loads, cytological analysis, and cytokines secretion in the lungs were evaluated at 6 or $24 \mathrm{~h}$ post infection with PAO1.

Intranasal priming with both lactobacilli blends significantly improved 7-day mice survival from $12 \%$ for the control PAO1 group to 71 and 100\% for the two groups receiving L.rff and L.psb respectively. No mortality was observed for both control groups receiving either L.rff or L.psb. Additionally, the PAO1 lung clearance was significantly enhanced at $24 \mathrm{~h}$. A 2-log and 4-log reduction was observed in the L.fff + PAO1 and L.psb + PAO1 groups respectively, compared to the control PAO1 group. Significant reductions in neutrophil recruitment and proinflammatory cytokine and chemokine secretion were observed after lactobacilli administration compared to saline solution, whereas IL-10 production was increased.

Conclusions: These results demonstrate that intranasal priming with lactobacilli acts as a prophylaxis, and avoids fatal complications caused by Pseudomonas aeruginosa pneumonia in mice. These results were independent of in vitro anti-Pseudomonas aeruginosa activity on QS-dependent virulence factors. Further experiments are required to identify the immune mechanism before initiating clinical trials.
\end{abstract}

Keywords: Lactobacillus, Probiotics, Intranasal administration, Pseudomonas aeruginosa, Respiratory tract infection, Mice

\footnotetext{
* Correspondence: rozenn.leberre@chu-brest.fr

${ }^{2}$ Univ Brest, Inserm, EFS, UMR 1078, GGB, Brest, France

${ }^{5}$ Département de Médecine Interne et Pneumologie, CHRU La Cavale Blanche, Brest, France

Full list of author information is available at the end of the article
}

(c) The Author(s). 2021 Open Access This article is licensed under a Creative Commons Attribution 4.0 International License, which permits use, sharing, adaptation, distribution and reproduction in any medium or format, as long as you give appropriate credit to the original author(s) and the source, provide a link to the Creative Commons licence, and indicate if changes were made. The images or other third party material in this article are included in the article's Creative Commons licence, unless indicated otherwise in a credit line to the material. If material is not included in the article's Creative Commons licence and your intended use is not permitted by statutory regulation or exceeds the permitted use, you will need to obtain permission directly from the copyright holder. To view a copy of this licence, visit http://creativecommons.org/licenses/by/4.0/. The Creative Commons Public Domain Dedication waiver (http://creativecommons.org/publicdomain/zero/1.0/) applies to the data made available in this article, unless otherwise stated in a credit line to the data. 


\section{Background}

Pulmonary infection with Pseudomonas aeruginosa in patients with cystic fibrosis (CF) and chronic obstructive pulmonary disease, is characterized by high morbidity and mortality $[1,2]$. Recently, the prevalence of $P$. aeruginosa community-acquired pneumonia among those with chronic lung disease and already colonised with $P$. aeruginosa was $67 \%$ [3]. In parallel, antibiotic resistance is increasingly leading to therapeutic deadlock. The European Centre for Disease Prevention and control found that in 2019, $12.1 \%$ of $P$. aeruginosa invasive isolates were resistant to at least three main antimicrobial groups [4]. In the USA, multidrug resistant $P$. aeruginosa account for 32,600 cases of infection and 2700 deaths [5].

Many antibiotic alternatives, such as probiotics, have been tested. Probiotics are defined as living microorganisms which, when administered in adequate amounts, confer a health benefit to the host [6]. Lactobacillus spp., the most studied probiotic, can be isolated from food and human mucosa. Their beneficial effect operates through different means, especially immunomodulatory and antibacterial activities [6]. Exploring their interaction with $P$. aeruginosa is difficult and could not be evaluated through bactericidal activity. Indeed, lactobacilli produce acids that directly inhibited the growth of $P$. aeruginosa [7]. Few studies tried however to explore their antipathogenic abilities in vitro against $P$. aeruginosa and focused on their impact against $P$. aeruginosa virulence factors [7-9]. Several strains have been shown to harbour anti-elastase and anti-biofilm properties [79]. In vivo, only two studies evaluated on murine model of pneumonia the beneficial effect of lactobacilli administration against $P$. aeruginosa $[10,11]$. Khailova et al. highlighted that oral administration of L. rhamnosus GG improves 7-day survival following $P$. aeruginosa-induced pneumonia. It is thought that regulatory $\mathrm{T}$ cells may play a role in this protection [11]. To date in man, randomized trials suggest that probiotics decrease the incidence of ventilator acquired pneumonia [12]; to counteract the many biases reported in these studies, a large randomized control trial is recruiting (PROSPECT study ClinicalTrials.gov Identifier: NCT02462590) [13].

While the oral route is often studied to analyse lactobacilli effects, the nasal route could provide benefits for respiratory infections by stimulating the activation of the lung immune response. Accordingly, studies show that intranasal administration of various Lactobacillus strains decrease mortality in viral pneumonia murine models $[14,15]$. Recently, we conducted the first study that investigates the protective effect of intratracheally inoculated lactobacilli against $P$. aeruginosa acute pneumonia on a mouse model [16]. It highlighted that the respiratory route of Lactobacillus administration may prevent from $P$. aeruginosa infection by decreasing the bacterial lung load and modulating the cytokine levels. Lactobacilli used have been mainly isolated from diaries. We hypothesized that clinical lactobacilli strains could be more adapted to the lung, than other probiotics strains administrated by oral route and usually isolated from the gut or diaries.

In this study, in the light of our previous results, we'd like first to screen for new lung-adapted probiotic strains to consider for in vivo studies, and second to confirm the use of the respiratory route for their administration against $P$. aeruginosa lung infection. The screening involved Lactobacillus isolates that we previously obtained from CF expectorations [17]. It was based on their capacity to inhibit two $P$. aeruginosa QS-dependent virulence factors (elastase and pyocyanin). We then evaluated the effect of intranasal administration of two blends of three selected lactobacilli strains on $P$. aeruginosa murine acute pneumonia. Primary outcomes were the survival and bacterial lung load $24 \mathrm{~h}$ after $P$. aeruginosa induced pneumonia. To decipher the effects of lactobacilli, cytological analysis, chemokines, and cytokine secretions from bronchoalveolar lavage (BAL) were measured.

\section{Results \\ In vitro screening of lactobacilli isolated from CF respiratory samples}

Forty strains (80\%) exhibited anti-elastolytic activity (mean activity $=-37.4 \% \pm 0.15)$, and $12(24 \%)$ exhibited anti-pyocyanin activity (mean activity $=-18.13 \% \pm 0.15$ ) (S1 Figure).

To constitute 2 blends of lactobacilli to administrate to the mice model of $P$. aeruginosa pneumonia, 3 strains with the highest anti-P. aeruginosa QS dependent virulence factor activities (L.rff) and 3 strains with no anti- $P$. aeruginosa QS dependent virulence factor activities (L.psb) were selected (S2 Table).

L.rff was constituted with $2 \mathrm{~L}$. fermentum strains and 1 L. rhamnosus strain (L. rhamnosus $2 C$, L. fermentum 9C and L. fermentum 10C).

L.psb was constituted with $1 \mathrm{~L}$. paracasei, $1 \mathrm{~L}$. salivarius and $1 \mathrm{~L}$. brevis strain (L. paracasei $9 N$, L. salivarius $20 C$ and L. brevis 24C).

Nasal priming with lactobacilli enhances the survival rate C57BL/6 mice were inoculated intranasally with each blend of lactobacilli $18 \mathrm{~h}$ prior to PAO1 administration (S3 Figure). All but two control PAO1 mice died (12\% survival). Mice receiving L.rff responded with $71 \%$ survival $(p<0.001)$. Mice receiving L.psb were fully protected (100\% survival) $(p<0.001$ compared to PAO1 group and $p<0.05$ compared to L.rff + PAO1 group). None of the control L.rff or control L.psb mice died or exhibited any clinical signs of distress (Fig. 1a). 

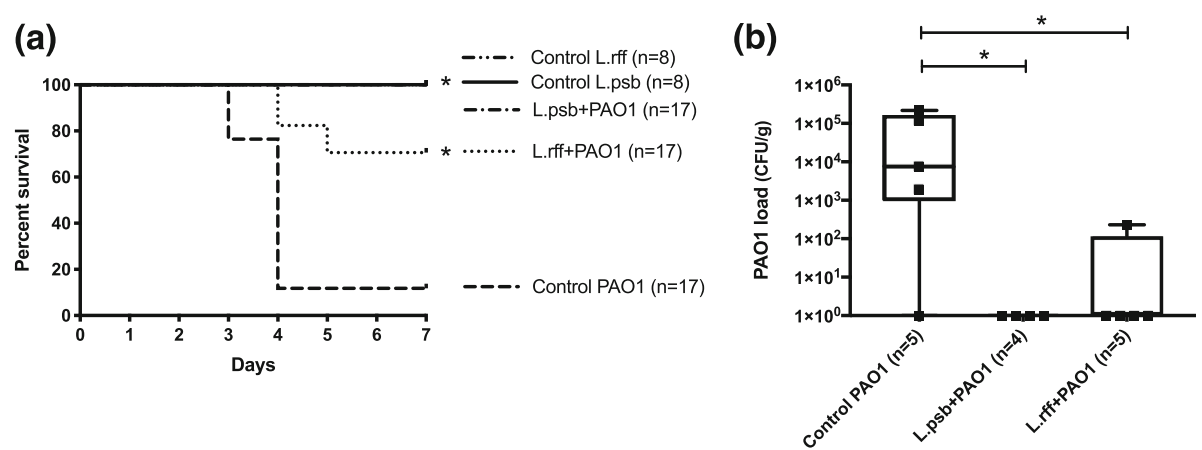

Fig. 1 a) Mice survival rate. Priming of the respiratory tract with L.psb $\left(1 \times 10^{6} \mathrm{CFU} / \mathrm{mouse}\right)$ or L.rff $\left(6 \times 10^{6} \mathrm{CFU} / \mathrm{mouse}\right)$ resulted in survival in response to $P$. aeruginosa infection $\left(6 \times 10^{6} \mathrm{CFU} /\right.$ mouse). Statistical significance: ${ }^{*}, p<0.001$ for the L.rff + PAO1 and L.psb + PAO1 groups compared to the control PAO1 group, and $p<0.05$ for the L.rff + PAO1 group compared to L.psb + PAO1 group. b) Pulmonary P. aeruginosa burden measured on total lung homogenates. Priming of the respiratory tract with L.psb $\left(2 \times 10^{5} \mathrm{CFU} / \mathrm{mouse}\right)$ or L.rff $\left(4 \times 10^{6} \mathrm{CFU} / \mathrm{mouse}\right) 18 \mathrm{~h}$ prior to $P$. aeruginosa infection $\left(6 \times 10^{6} \mathrm{CFU} /\right.$ mouse) enhanced the clearance of $P$. aeruginosa. Statistical significance: ${ }^{*}, p<0.05$ compared to the control PAO1 group

\section{Administration of lactobacilli decreases the lung $P$. aeruginosa load}

After $24 \mathrm{~h}$ a 2 -log and 4-log reduction was observed in the L.rff + PAO1 and L.psb + PAO1 groups respectively, compared to the control PAO1 group $(p<0.001$, Fig. 1b).

No increase in lactobacilli load was observed in either group. However, lactobacilli were still present in the lung $24 \mathrm{~h}$ after the instillation, with $1 \times 10^{4}$ and $1 \times 10^{3}$ $\mathrm{CFU} / \mathrm{g}$ for the L.rff + PAO1 and L.psb + PAO1 groups respectively.

White blood cell count and cytokine analysis in BAL fluid To elucidate the mechanism of the $P$. aeruginosa lung load reduction, we investigated the white blood cell count (WBC) recruitment and cytokine synthesis in the BAL fluid (Fig. 2).

No difference was observed in the WBC at T6 and T24 between the control L.rff group and the control sham group. However, qualitatively, the BAL fluid infiltrate was mostly composed of polymorphonuclear (PMN) in the control L.rff group at T6 whereas the BAL fluid of sham mice only included alveolar macrophages (AM). As expected, mice from the control PAO1 group exhibited a strong increased number of WBC, which was mainly composed of neutrophils. This recruitment is more significant ( 3 and 9 times more respectively at T6 and T24) than in the control L.rff mice. A significant decrease of PMN in BAL fluid was observed at T6 and T24 in the L.rff + PAO1 and L.psb + PA01 groups compared to the PAO1 group.

We investigated the immunological response due to prophylactic administration of lactobacilli through proinflammatory cytokine and chemokine dosage in the BAL fluid. The anti-inflammatory cytokine IL-10 was also evaluated.
Administration of lactobacilli alone did not induce the secretion of CXCL1, CXCL2, IL-1 $\beta$, IL- 6 and TNF- $\alpha$ compared to sham mice. Infection of PAO1 induced a cytokine burst particularly at $6 \mathrm{~h}$. Prophylactic administration of lactobacilli leads to lower secretions of chemokines CXCL1 and CXCL2 (at T6) and proinflammatory cytokines IL-1 $\beta$, IL- 6 and TNF- $\alpha$ (both at T6 and T24) in both L.rff + PAO1 and L.psb + PA01 groups compared to the control PAO1 group (Figs. 3 and 4). Whereas PAO1 infection tends to decrease the IL-10 levels in the BAL, its production was significantly increased in the L.psb group compared to the control PAO1 groups (Fig. 5) but no difference was observed with the sham group.

\section{Discussion}

In this study, intranasal administration of lactobacilli in a murine model of $P$. aeruginosa pneumonia significantly improved lung $P$. aeruginosa clearance $24 \mathrm{~h}$ post infection, and prevented mice death. This prophylactic treatment was associated with a lower secretion of chemokines and proinflammatory cytokines, and a lower neutrophil recruitment. This shows the implication of the innate immune response in this mechanism.

Pulmonary effects of Lactobacillus administration have been explored through oral gavage [10,11, 18-22] or intranasal administration $[14,15,23-26]$ and mainly focused on virus pathogens or S. pneumoniae. Oral gavage suggests that the protective abilities of Lactobacillus are based on the immunomodulation mediated by the gutlung axis. Indeed, the gut microbiota interferes with the immune system, notably with granulocytosis by priming it through commensal components and pathogenic microorganisms which activate pattern recognition receptors (PRR) [27]. This reverberates on the lung and thus, any modification of the gut microbiota with oral 

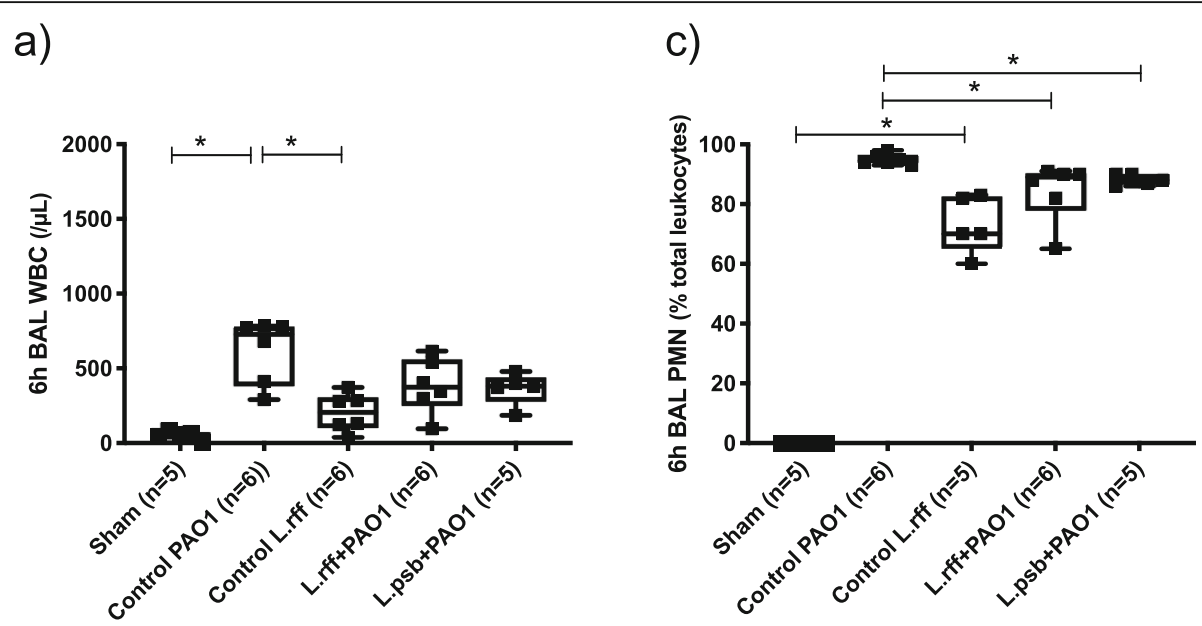

b)

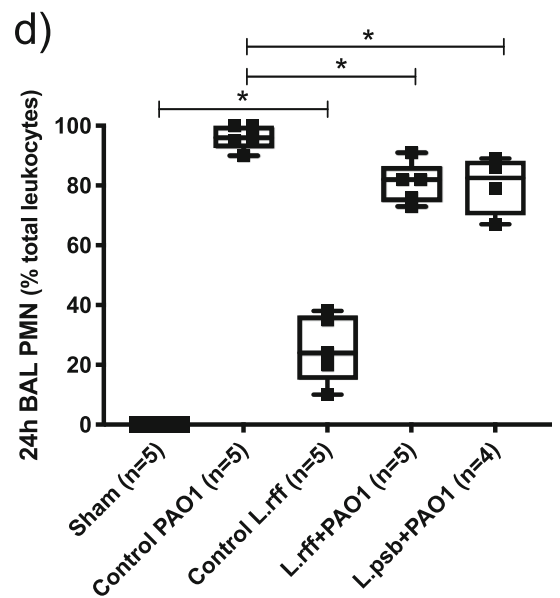

Fig. 2 a) and b) Total white blood cell count in BAL fluids at $6 \mathrm{~h}$ and $24 \mathrm{~h}$ post infection with P. aeruginosa. c) and d) Polymorphonuclear cell ratio in BAL fluids at $6 \mathrm{~h}$ and $24 \mathrm{~h}$ post infection with $P$. aeruginosa. Statistical significance: *, $p<0.05$; BAL, Bronchoalveolar lavage; PMN, Polymorphonuclear cells; WBC, White blood cells

administration of probiotics may influence lung immunity at distance. The lung microbiota may also directly interfere with the immune system through AM and dendritic cells which express various PRR. In our study, lactobacilli were administered intranasally in order to act directly on the airway mucosa immune system and to bypass the gut-lung axis (although swallowing cannot be totally excluded). We expected an enhanced, faster, and more appropriate response compared to the one following oral gavage. For the first time, our data showed that lactobacilli could sufficiently stimulate the respiratory mucosal immune system in situ to protect from P. aeruginosa infection. This approach already highlighted interesting results notably when used as a vaccine, against the flu or S. pneumoniae [28, 29]. This way of administration is otherwise safe for the mice, associated with a low number of PMN recruitment and absence of antiLactobacillus antibody secretion [24, 30, 31].

To our knowledge, our study is the first to explore the effects of intranasal administration of lactobacilli against
$P$. aeruginosa respiratory infection. We observed an enhancement of lung $P$. aeruginosa clearance at $24 \mathrm{~h}$ post infection, associated with the decrease of chemokines and proinflammatory cytokines, the restoration of IL-10 and a reduction in neutrophil recruitment. The host immune response against acute $P$. aeruginosa infection is mainly based on the PMNs recruitment and activation, as neutropenic mice lead to higher $P$. aeruginosa mortality [32]. The PMNs recruitment is triggered secondly to $P$. aeruginosa interaction with AM or the airway epithelium, and through the toll like receptor signalling pathway [33]. However, an excess of PMNs was not always effective to clear the bacteria and caused serious tissue damage. A favourable outcome to $P$. aeruginosa infection is based on the adapted PMN recruitment, which allows for survival by clearance of the pathogen without excessive inflammation. The regulation of the inflammatory response is mainly based on anti-inflammatory cytokines, notably the IL-10 [34]. In our study, lactobacilli administered intranasally seemed to act on the innate 


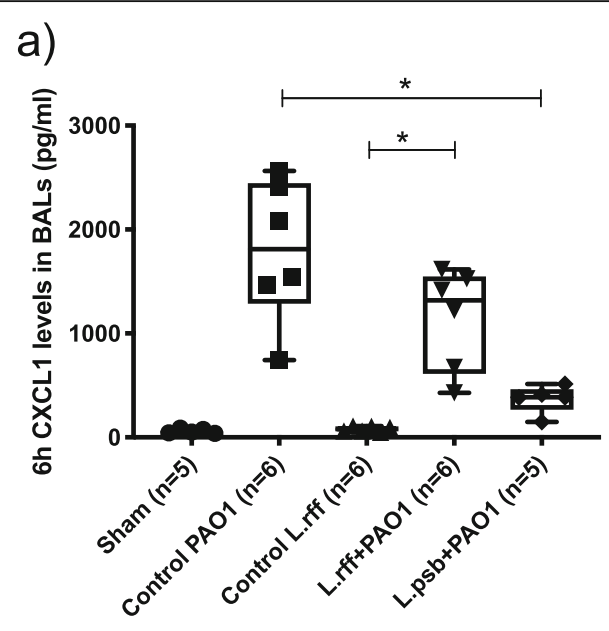

b)

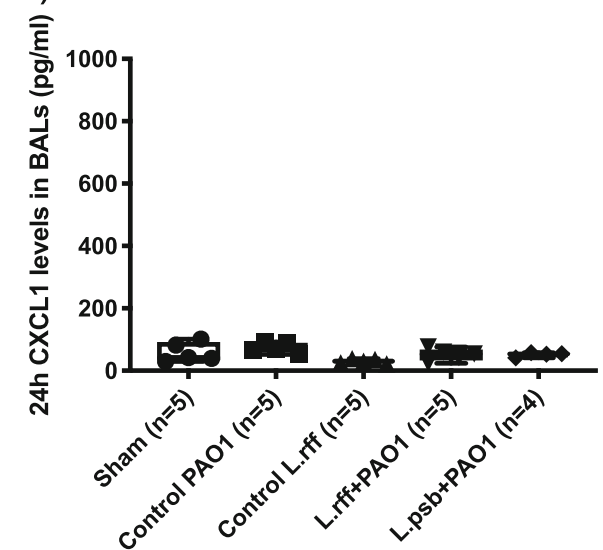

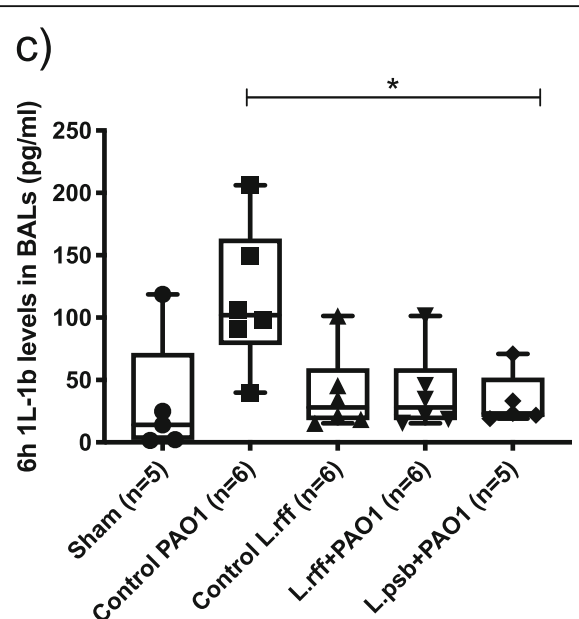

d)

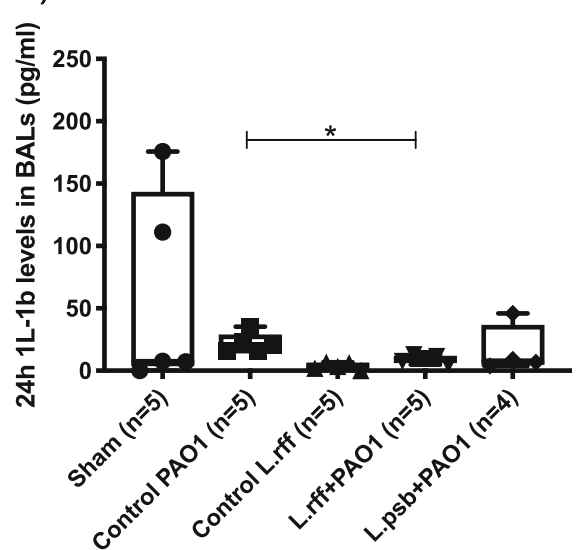

Fig. 3 a) and b) CXCL1 levels in BALs at $6 \mathrm{~h}$ and $24 \mathrm{~h}$ post infection with $P$. aeruginosa. c) and $\mathbf{d}$ ) IL-1b levels in BAL fluids at $6 \mathrm{~h}$ and $24 \mathrm{~h}$ post infection with $P$. aeruginosa. Statistical significance: ${ }^{*}, p<0.05$; BAL, Bronchoalveolar lavage

immunity, particularly on PMNs. Indeed, PMNs were more significantly and transiently recruited in the control L.rff group compared to sham mice, with no consequence on the mortality, suggesting a beneficial preventive PMNs recruitment. After $P$. aeruginosa infection, the priming resulted in a reduced inflammatory response triggered by $P$. aeruginosa, and attenuated the recruitment of PMNs through a decreased secretion of proinflammatory cytokines and chemokines. Even with less PMNs, the P. aeruginosa clearance was enhanced, and the survival improved. Antibacterial effect of lactobacilli might be related to a priming of resident effector cells such as alveolar macrophages. Indeed, recent reports showed that lactobacillus strains are able to activate macrophages and to promote phagocytic and bactericidal activity in macrophages [35, 36]. Some previous studies focusing on priming with probiotics against various pathogens mostly agreed that probiotics could beneficially regulate the balance between pro and antiinflammatory cytokines $[18,20,25]$. Others studies focusing on $P$. aeruginosa using the oral route to administer lactobacilli highlighted interesting results. According to Khailova and colleagues, the adaptive immunological response may be involved through regulatory $\mathrm{T}$ cells [11]. Alvarez and colleagues implicate the innate response, through an enhancement of the phagocytic activity of AM, associated with the activation of the specific response through secretion of IgA and IgM in the BAL fluid [10]. However, they didn't observe any difference in white blood cell differential counts, confirming the interest of our work. In our study, we did not observe any lymphocyte recruitment following the administration of lactobacilli. However, we used different strains, means of administration, and mice. Our results may also implicate the innate response, through the modulation of the phagocytic activity conducted by decreased proinflammatory cytokines and chemokines, and increased IL-10. The $P$. aeruginosa clearance led by lactobacilli may be based on the modulation of the bactericidal and phagocytic activities of PMNs. The use of neutropenic mice may help to clarify the exact mechanism. 
a)

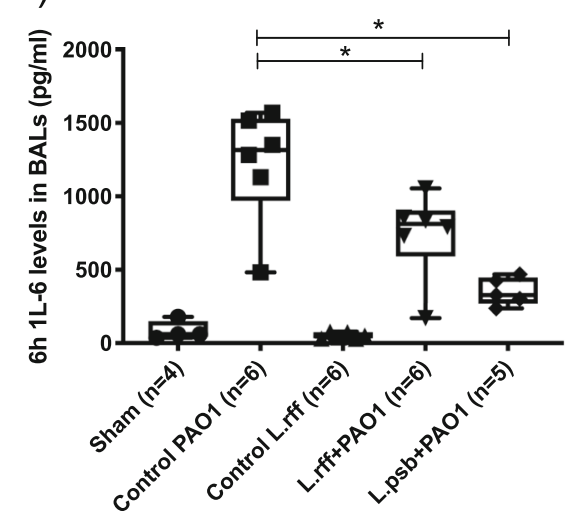

b)

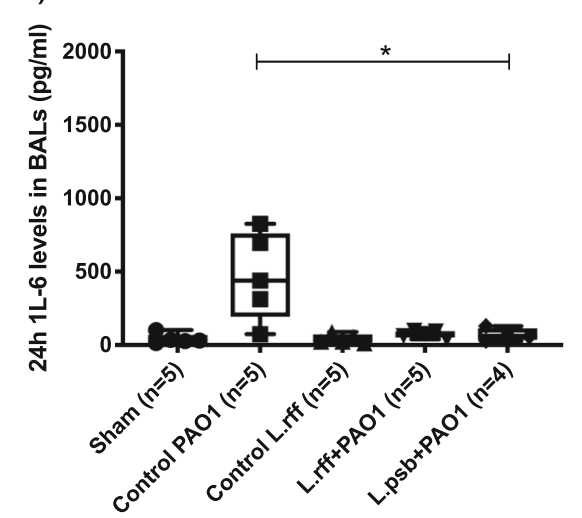

c)

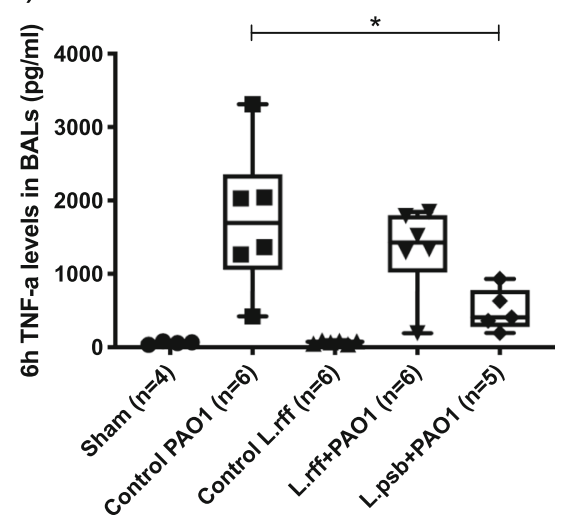

d)

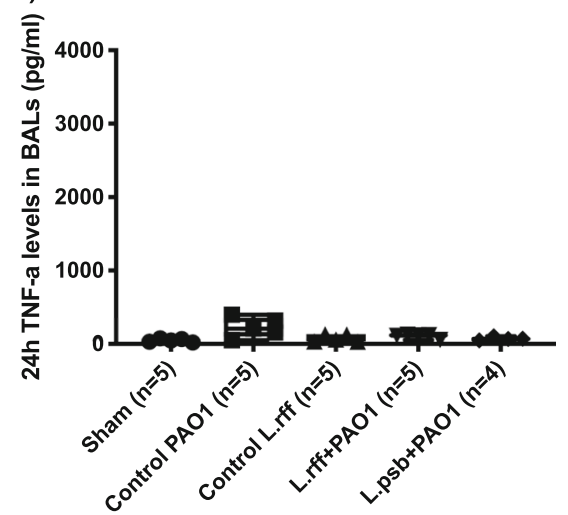

Fig. 4 a) and b) IL-6 levels in BAL fluids at $6 \mathrm{~h}$ and $24 \mathrm{~h}$ post infection with P. aeruginosa. c) and d) TNF-a levels in BAL fluids at $6 \mathrm{~h}$ and $24 \mathrm{~h}$ post infection with $P$. aeruginosa. Statistical significance: ${ }^{*}, p<0.05$; BAL, Bronchoalveolar lavage
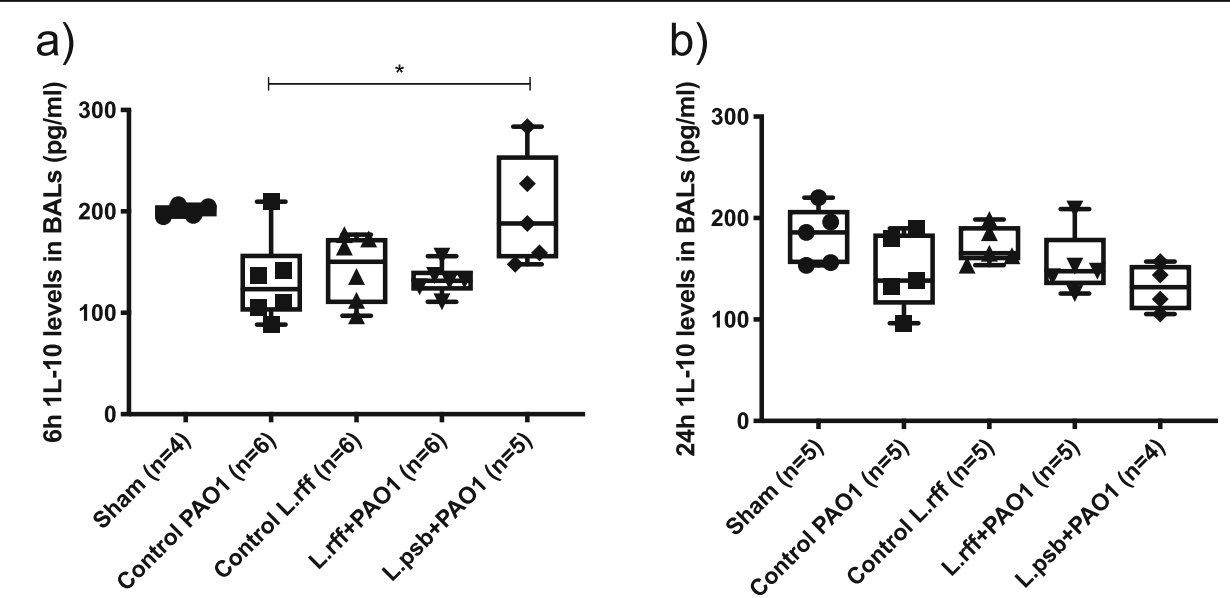

Fig. 5 a) and b) IL-10 levels in BAL fluids at $6 \mathrm{~h}$ and $24 \mathrm{~h}$ post infection with $P$. aeruginosa. Statistical significance: *, $p<0.05$. BAL, Bronchoalveolar lavage 
Lactobacilli have a long history of safe use by oral route [37] but the safety of lactobacilli application in the respiratory tract is crucial and needs to be studied. The longstanding dogma that the normal lung is free from bacteria [38] has been overturned by the recent advent of culture-independent techniques of microbial identification. Several publications showed that healthy human lungs contain diverse bacterial communities [39-41]. Additionally, in a recent study, Rosas-Salazar et al. [42] highlighted that lactobacilli was one of the 35 most abundant genus in the nasopharyngeal microbiome in infancy and showed a protective effect of upper airway colonization with Lactobacillus on asthma-related outcomes in children. Even with encouraging survival data, it will be necessary to analyse if the administration of Lactobacillus isolates in the respiratory tract lead to lung microbiota modifications, and to a particular microbiota signature already described in pulmonary diseases [43]. If Lactobacillus candidates are identified on the basis of convincing animal data, clinical trials phase I, II and III in humans will be essential to determine their safety.

$P$. aeruginosa pneumonia is still a lethal infection despite the use of antibiotics, especially among intensive care unit patients or patients with chronic respiratory diseases such as cystic fibrosis. There is an urgent need to develop alternative treatments to antibiotic therapy. Probiotics have demonstrated their capability to protect from pathogens through heterologous immunity. Several studies on the murine model of pneumonia highlighted that the administration of Lactobacillus could crossprotect from a later viral or bacterial infection $[10,11$, $15,18,22,23,26]$. Furthermore, their abilities to protect humans from pulmonary infections have also been demonstrated [6]. The heterologous immunity is thus expanding as a therapeutically strategy [44]. Various Lactobacillus strains are used to prevent respiratory infections, with probiotic activity being strain dependent $[11,45,46]$. Few authors tried to screen their own strains before experimenting [7, 21, 30, 31, 46]. Screening usually focuses on the strain's ability to inhibit virulence factors of the studied pathogen [7, 46, 47], to produce inhibitory substances [30,46] or cytokines [21], or to select the strains with the better adhesive properties in their experiment models $[31,46]$. In our study, we screened for new lung-adapted probiotic strains with anti- $P$. aeruginos $a$ abilities. This screening was based on a previous work, which studied the prevalence of lactobacilli in the lung of CF patients in regard to their $P$. aeruginosa colonisation status [17]. As no difference in species diversity or frequency was observed, we secondly screened a representative sample of 50 strains for their in vitro ability to inhibit two virulence factors produced by $P$. aeruginosa, the elastase and pyocyanine. These two major virulence factors are regulated by quorum sensing, cause a wide range of pathogenic effects and extensive tissue damage, and their pathogenicity has been confirmed in vivo on murine models of $P$. aeruginosa pneumonia [48, 49]. Furthermore, this screening strategy is based on the inhibition of two virulence factors after neutralising to $\mathrm{pH} 7$ the coculture. This avoids the direct antibacterial effect due to Lactobacillus lactic and acetic acids production [7]. Our approach is original as we choose to highlight novel respiratory strains with great in vitro potential against $P$. aeruginosa. Despite thoughtful screening, improved survivals were observed whatever the blend of lactobacilli administered as a prophylactic treatment. Otherwise, the L.psb blend constituted with ineffective strains against $P$. aeruginosa QSdependent virulence factors in vitro seemed to better act in vivo against $P$. aeruginosa. These results will deserve to be confirmed with other experiments but already raised a number of interesting questions concerning the mechanisms of action underlying the beneficial effect observed against $P$. aeruginosa. Additional are also required in order to determine the kinetic of the beneficial effect of our blends and their activity during chronic infection. The analysis of the lung clearance of our blends should be conducted in parallel. Although we report that administration of L.rff blend did not alter the health status in naïve mice and induce a mild and transient neutrophil recruitment not associated with a lung cytokine burst, these data should be extended.

A limitation of our study is related to the lack of data concerning the safety of the L.psb treatment about cytokine dosages and cytological analysis. However, it may be extrapolated to L.rff control group results. Indeed, in our first set of experiment, we focused on the prophylactic activity of the lactobacilli cocktail with a strong in vitro activity (L.rff cocktail) that we compared with a cocktail without in vitro activity (L.psb cocktail). Thus we only included a control group receiving the L.rff blend. Since the L.psb cocktail with no inhibitory abilities against $P$. aeruginosa had a similar in vivo activity against PAO1 during this first set of experiment, we added one supplementary L.psb control group receiving this blend for survival assay to evaluate their safety.

This study suggests that the lactobacilli abilities in vitro cannot predict their in vivo abilities to fight against $P$. aeruginosa pneumonia. The two QS-dependent virulence factors, elastase and pyocyanin, probably do not interfere directly with the immune response in our murine model of $P$. aeruginosa pneumonia. Indeed, numerous $P$. aeruginosa-virulence factors have been identified but the contribution of each of them in lung pathogenicity is not deciphered. In addition, further experiments to elucidate the precise mechanism of action are needed. Notably, the priming mediated by each single strain should be studied in distinct experimentations. 


\section{Conclusions}

In summary, we screened in vitro 50 Lactobacillus strains on their ability to inhibit the synthesis of 2 P. aeruginosa PAO1 virulence factors. Two blends of three Lactobacillus strains were constituted and intranasally administrated in a mice model of murine acute pneumonia. Our results firstly showed that intranasal administration of Lactobacillus strains can prevent from $P$. aeruginosa acute pneumonia by enhancing the mice survival, and modulating their local lung immunity. Secondly, in vitro abilities cannot predict lactobacilli in vivo abilities, as both blend of Lactobacillus improved mice survival, even when Lactobacillus strains administered didn't diminished $P$. aeruginosa virulence factors production in vitro. The comprehension of the mechanisms involved in the immunomodulation requires further experimentation. The priming mediated by each single strain (alive or heat killed) of the promising L.psb cocktail (L. paracasei $9 \mathrm{~N}$, L. salivarius 20C, L. brevis 24C) should be studied in distinct in vivo experimentations.

\section{Methods \\ Inhibition tests of Lactobacillus strains on PAO1 on elastase and pyocyanin virulence factors}

To administer Lactobacillus in the respiratory tract of mice, we screen for Lactobacillus isolates well adapted to the lung for their abilities to fight against $P$. aeruginosa. As lactobacilli produce acids that directly inhibit $P$. aeruginosa, bactericidal assays cannot be used for screening [7]. Fifty Lactobacillus strains previously isolated from respiratory samples from patients with CF [17] were thus screened in vitro for their ability to decrease the synthesis of $2 P$. aeruginosa QSdependent virulence factors, pyocyanin and elastase. $P$. aeruginosa PAO1 was chosen as the reference strain for all experiments [50]. Samples were stored at $-80{ }^{\circ} \mathrm{C}$ prior to subculturing on Mueller Hilton agar plates $(\mathrm{MH})$ (bioMérieux) before experiments. All isolates were frozen at $-80{ }^{\circ} \mathrm{C}$ before subculture on $5 \%$ sheep-blood agar (bioMérieux) in $5 \% \mathrm{CO}_{2}$ at $37^{\circ} \mathrm{C} 2$ days before experiments.

Elastase: PAO1 and each Lactobacillus isolate were separately cultivated overnight at $37^{\circ} \mathrm{C}$ in Brain Heart Infusion broth (BHI) (Oxoïd) in $50 \mathrm{ml}$ Falcon conical tubes. The inhibition of the elastolytic activity of PAO1 by Lactobacillus isolates was investigated by colorimetric assay, using Elastin Congo Red (ECR) (Sigma) as adapted by Alexandre et al. [7]. Succinctly, overnight aerobic culture of PAO1 in BHI broth under agitation was washed twice with isotonic saline solution and adjusted to $5 \times$ $10^{7} \mathrm{CFU} / \mathrm{ml}$ in broth media. Overnight static culture of Lactobacillus in BHI broth was neutralised with $\mathrm{NaOH}$ $0,1 \mathrm{M}$ to $\mathrm{pH} 7$ and adjusted to $5 \times 10^{7} \mathrm{CFU} / \mathrm{ml}$ in broth media. A $2 \mathrm{ml}$ co-culture was made for each
Lactobacillus isolates, by transferring $1 \mathrm{ml}$ of the neutralised Lactobacillus broth to $1 \mathrm{ml}$ of the PAO1 broth ( $\mathrm{vol} / \mathrm{vol}$ ) and incubated in a tube under agitation $20 \mathrm{~h}$ under aerobic conditions at $37^{\circ} \mathrm{C}$. After centrifugation (20' at $3500 \mathrm{~g}$ ), $50 \mu \mathrm{l}$ of the supernatant was mixed with $1 \mathrm{ml}$ of Elastin Congo Red solution $(20 \mathrm{mg} / \mathrm{ml}$ in a 10 $\mathrm{mM}$ sodium phosphate buffer) in a $2 \mathrm{ml}$-Eppendorf tube and incubated for $20 \mathrm{~h}$ more with rotation. Finally, the soluble fraction released in the supernatant by elastase was measured at $495 \mathrm{~nm}$ after centrifugation $\left(20^{\prime}\right.$ at $3500 \mathrm{~g}$ ) in a microplate spectrophotometer (Multiskan FC Micro- plate Photometer, Thermo Scientific).

Pyocyanin: PAO1 was grown overnight in BactoPeptone (BP) broth (20 mg/l BP, $\mathrm{MgCl}_{2} 1,4 \mathrm{~g} / \mathrm{l}, \mathrm{K}_{2} \mathrm{SO}_{4}$ $10 \mathrm{~g} / \mathrm{l})$ (Oxoïd) and Lactobacillus isolates in MRS broth (bioMérieux). A $2 \mathrm{ml} \mathrm{vol} / \mathrm{vol}$ co-culture was made as previously described for the elastase experiments, and incubate under aerobic conditions at $37^{\circ} \mathrm{C}$. The inhibition of the pyocyanin synthesis was investigated by colorimetric assay after extraction as previously described by Schaber et al. [51]. After centrifugation (20' at 3500 g) of the co-culture, $50 \mu \mathrm{l}$ of the supernatant was mixed with $50 \mu \mathrm{l}$ of chloroform. The lower phase was transferred in a $15 \mathrm{ml}$ Falcon conical tube and mixed with 2 $\mathrm{ml}$ of $\mathrm{HCl}(0.2 \mathrm{M})$. Finally, the pyocyanin extracted in the organic layer was measured at $520 \mathrm{~nm}$ in a microplate spectrophotometer (Multiskan FC Micro- plate Photometer, Thermo Scientific).

Pyocyanin and elastase results were normalised according to the $\mathrm{OD}_{595}$ of the co-culture and expressed as a ratio of the absorbance observed in presence of the Lactobacillus isolate to the absorbance observed with a monoculture of PAO1. Two experiments were conducted independently.

\section{Preparation of the bacterial strains}

As commercial probiotics preparations are often made of several strains, a blend was constituted for the murine model of pneumonia. Three strains with the highest inhibitory abilities against PAO1 virulence factors were equally mixed in a blend named "L.rff" (composed of $L$. rhamnosus 2C, L. fermentum 9C and L. fermentum 10C). Three strains without inhibitory activity were mixed as a control in a blend named "L.psb" (L. paracasei $9 N, L$. salivarius $20 C$ and L. brevis 24C). Lactobacilli were grown overnight in MRS broth under aerobic conditions at $37^{\circ} \mathrm{C}$.

$P$. aeruginosa PAO1 was chosen as the reference strain for all experiments [50]. Samples were stored at $-80{ }^{\circ} \mathrm{C}$ prior to subculturing on Mueller Hilton agar plates (MH) (bioMérieux) before experiments. Then, PAO1 was grown overnight in Luria-Bertani broth (Sigma) under aerobic conditions at $37^{\circ} \mathrm{C}$. Each culture was washed twice with isotonic saline solution (SS) and 
adjusted to $10^{9} \mathrm{CFU} / \mathrm{ml}$ for the PAO1 suspension, or to $10^{7} \mathrm{CFU} / \mathrm{ml}$ for the L.rff and L.psb suspensions, based on the $\mathrm{OD}_{595 \mathrm{~nm}}$ and controlled by serial dilution and plating on $\mathrm{MH}$ in triplicates.

\section{Murine model of acute pneumonia}

This study (APAFIS\#9623-2,017,040,717,237,994 and APAFIS\#12025-2,017,110,311,134,961) has been approved by the french ethics committee for animal experiments $\mathrm{n}^{\circ} 074$ under the responsibility of the french ministry of higher education and research.

C57BL/6 J mice, aged 6-8 weeks old, were purchased from Janvier Labs (Le Genest Saint Isle, France) and maintained at the University of Brest, France. The mice were maintained in constant temperature $\left(22^{\circ} \mathrm{C}\right)$ and environment humidity room. Mice were fed ad libitum and monitored every eight hours until being sacrificed. All mice received human care.

For the cytokine dosages, cytological and bacterial burden experiments, mice were randomly assigned to 5 groups ( $n=5$ to 6): Sham mice, Control PAO1, Control L.rff and study group PAO1 + L.rff. A supplementary study group PAO1 + L.psb was add as a control of the PAO1 + L.rff group. The aim was to compare in vivo the effects conferred by the administration of three Lactobacillus strains with and without inhibitive properties against elastase and pyocyanin synthesis.

For the survival experiments, considering the results obtained from cytokine dosages, cytological and bacterial burden experiments, we add one more supplementary control Lactobacillus group named Control L.psb. Thus, 67 mice were randomly assigned to 5 groups $(n=8$ for control L.rff and L.psb groups and $n=17$ for Control PAO1, PAO1 + L.psb and PAO1 + L.rff groups).

The control mice (sham) received isotonic saline solution. Mice were intranasally inoculated with $20 \mu \mathrm{l}$ of the bacterial suspension $(10 \mu \mathrm{l}$ per nostril), under intraperitoneal anaesthesia with ketamine/xylazine $(100 / 10 \mathrm{mg} / \mathrm{kg})$.

Lactobacilli suspension (L.rff or L.psb) was administered $18 \mathrm{~h}$ prior to infection with PAO1. The control PAO1 group received SS instead of lactobacilli. The L.rff and L.psb control groups received SS instead of PAO1.

Six (T6) or $24 \mathrm{~h}$ (T24) post infection with PAO1, mice were anesthetized with intraperitoneal injection of ketamine/xylazine $(100 / 10 \mathrm{mg} / \mathrm{kg})$ and sacrificed by intracardiac exsanguination. BAL was performed after euthanasia by cannulation of the trachea and injection and aspiration of $500 \mu \mathrm{l}$ of SS three times.

Blood, BAL fluid, lung and spleen tissues were harvested from animals under aseptic conditions.

\section{Bacterial burden in lung homogenates}

Mice were sacrificed at T24 and lungs removed and homogenized with SS with Ultra-Turrax. Bacterial loads of
PAO1 and Lactobacillus blends were determined by plating serial dilutions of total lung homogenate on Cetrimide (bioMérieux) and MRS agar plates. Each dilution was plated in duplicate. Plates were incubated $24 \mathrm{~h}$ to $48 \mathrm{~h}$ at $37^{\circ} \mathrm{C}$ under aerobic conditions. The limit of detection by platting was $40 \mathrm{CFU} / \mathrm{ml}$. Colonies were identified using MALDI-TOF mass spectrometry (Microflex LT, Bruker Daltonics, Bremen, Germany).

\section{White blood cell count}

The total white blood cell (WBC) count on BAL fluid was enumerated by a manual counting method with a hemocytometer (Kova slide) by light microscopy.

Alveolar macrophages (AM), polymorphonuclear cells (PMNs) and lymphocytes were differentiated after centrifugation, cytospins preparation and May-GrünwaldGiemsa staining.

\section{Cytokine measurement on BAL fluid}

The cytokines studied were IL-1 $\beta$, IL-6, IL-10, TNF- $\alpha$, and the 2 chemokines CXCL- 1 and CXCL-2. IL-1 $\beta$, IL- 6 and IL-10 (eBiosciences), TNF- $\alpha$ and the chemokines CXCL1 and CXCL2 (R\&D System, Abingdon, UK) were determined in the BAL fluid by enzyme-linked immunosorbent assay (ELISA), using commercial kits according to the manufacturer's recommendations. The lower levels of detection were $7 \mathrm{pg} / \mathrm{ml}$ for CXCL1 and CXCL2, $4 \mathrm{pg} / \mathrm{ml}$ for IL- $1 \beta$ and IL- $6,8 \mathrm{pg} / \mathrm{ml}$ for IL- 10 and TNF$\alpha$.

\section{Survival experiments}

Mice were monitored during 7 days after infection with PAO1. Fur aspect, activity, behavior, posture, eyelids, respiration, chest sounds, and body weight were followed frequently during the whole experiment, and scored from 1 to 4 according to the M-CASS scoring system [52]. When mice reached a total score of 11 during the day, buprenorphine was administered subcutaneously $(0.05 \mathrm{mg} / \mathrm{kg} / 12 \mathrm{~h})$ for analgesia. Mice were sacrificed when they reached a score of 4 in the 8 parameters during the day, or in one parameter at night to prevent overnight death.

\section{Statistics}

Results are presented as a boxplot. Comparisons between the groups were analysed by the Mann-Whitney test. The analysis of survival was performed with the Kaplan-Meier method. Results were considered statistically significant for $p<0.05$. All statistical tests were performed using the R software.

\section{Abbreviations}

AM: Alveolar macrophages; BAL: Bronchoalveolar lavage; BHI: Brain heart infusion; CF: Cystic fibrosis; CFU: Colony forming unit; $\mathrm{MH}$ : Mueller Hilton 
agar plate; OD: Optical density; PRR: Pattern recognition receptors;

PMN: Polymorphonuclear; SS: Isotonic saline solution

\section{Supplementary Information}

The online version contains supplementary material available at https://doi. org/10.1186/s12866-021-02254-7.

Additional file 1: S1 Figure. Anti-PA activities of the 50 Lactobacillus strains screened. S2 Table. Anti-PA activities of the 6 Lactobacillus strains selected to be administered to the mice. S3 Figure. Study design. S4 Table. ARRIVE Essential 10 checklist for animal research and Recommended Set of the National Centre for the Replacement Refinement and Reduction of Animals in Research.

\section{Acknowledgements}

We would like to thank Kristin McHale for the English proofreading. We would like to thank the entire team of the animal facility. We would like to thank VLM (Vaincre La Mucoviscidose) association.

\section{Authors' contributions}

Conceived and designed the study: MSF, RLB, PG. Collected the data: MSF, PG, NG, SG. Performed the analysis: MSF, RLB, PG, NG, SG, CAG. Wrote the first draft: MSF, PG, RLB. Reviewed the paper: MSF, PG, RLB, CP, SV, GHA and gave final approval: All the authors.

\section{Funding}

This research did not receive any specific grants from funding agencies in the public, commercial, or not-for-profit sectors.

\section{Availability of data and materials}

The datasets used and/or analysed during the current study are available from the corresponding author on reasonable request.

\section{Declarations}

\section{Ethics approval and consent to participate}

The Université de Bretagne Occidentale's animal facility works under the European Directive 2010/63/UE of the Council of Europe on Animal Welfare and its French transposition (European Directive 2010/063 EU, French « Decree $n^{\circ}$ 2013-118 » about protection of animals used for scientific purposes). Animal facility has the official license number D29-019-04 delivered by the official regional vet (DDPP) to take care of animals, breed them and perform protocols.

In accordance with the directive 2010/63/UE, our projects have been submitted to the French "Comité d'Ethique Finistérien en Expérimentation Animale" number 74 (CEFEA-74, cefea@univ-brest.fr) on which our university depends under the responsibility of the french ministry of higher education and research. We received autorisation numbers APAFIS\#96232017040717237994 and APAFIS\#12025-2017110311134961.

The ARRIVE essential 10 checklist for animal research and the Recommended set of the National Centre for the Replacement Refinement and Reduction of Animals in Research have been completed to assess the reliability of the findings (\$4 Table.).

\section{Consent for publication}

Not applicable.

\section{Competing interests}

All authors have no interests to declare.

\section{Author details}

${ }^{1}$ Laboratoire de biologie médicale, Centre Hospitalier de Cornouaille, Quimper, France. ${ }^{2}$ Univ Brest, Inserm, EFS, UMR 1078, GGB, Brest, France. ${ }^{3}$ University of Lille, CNRS UMR9017, Inserm U1019, CHRU Lille, Institut Pasteur de Lille, CIIL - Center for Infection and Immunity of Lille- OpInfIELD, Lille, France. ${ }^{4}$ Département de Bactériologie-Virologie, Hygiène hospitalière et Parasitologie-Mycologie, CHRU La Cavale Blanche, Brest, France. ${ }^{5}$ Département de Médecine Interne et Pneumologie, CHRU La Cavale Blanche, Brest, France.
Received: 27 July 2020 Accepted: 9 June 2021

Published online: 28 June 2021

\section{References}

1. Langan KM, Kotsimbos T, Peleg AY. Managing Pseudomonas aeruginosa respiratory infections in cystic fibrosis. Curr Opin Infect Dis. 2015;28(6): 547-56.

2. Fujitani S, Sun HY, Yu VL, Weingarten JA. Pneumonia due to Pseudomonas aeruginosa: part l: epidemiology, clinical diagnosis, and source. Chest. 2011; 139(4):909-19.

3. Restrepo MI, Babu BL, Reyes LF, Chalmers JD, Soni NJ, Sibila O, et al. Glimp. Burden and risk factors for Pseudomonas aeruginosa community-acquired pneumonia: a multinational point prevalence study of hospitalised patients. Eur Respir J. 2018;52:1701190.

4. European Centre for Disease Prevention and Control (ECDC). Antimicrobial resistance surveillance in Europe 2019. In: Annual report of the European antimicrobial resistance surveillance network (EARS-net). Stockholm: ECDC; 2020.

5. CDC. Antibiotic Resistance Threats in the United States, 2019. Atlanta: U.S. Department of Health and Human Services; 2019.

6. Alexandre Y, Le Blay G, Boisrame-Gastrin S, Le Gall F, Hery-Arnaud G, Gouriou S, et al. Probiotics: a new way to fight bacterial pulmonary infections? Med Mal Infect. 2014;44(1):9-17.

7. Alexandre Y, Le Berre R, Barbier G, Le Blay G. Screening of Lactobacillus spp for the prevention of Pseudomonas aeruginosa pulmonary infections. BMC Microbiol. 2014:14:107

8. Valdez JC, Peral MC, Rachid M, Santana M, Perdigon G. Interference of Lactobacillus plantarum with Pseudomonas aeruginosa in vitro and in infected burns: the potential use of probiotics in wound treatment. Clin Microbiol Infect. 2005;11(6):472-9.

9. Ramos AN, Cabral ME, Noseda D, Bosch A, Yantorno OM, Valdez JC. Antipathogenic properties of Lactobacillus plantarum on Pseudomonas aeruginosa: the potential use of its supernatants in the treatment of infected chronic wounds. Wound Repair Regen. 2012;20(4):552-62.

10. Alvarez S, Herrero C, Bru E, Perdigon G. Effect of Lactobacillus casei and yogurt administration on prevention of Pseudomonas aeruginosa infection in young mice. J Food Prot. 2001;64(11):1768-74.

11. Khailova L, Baird CH, Rush AA, McNamee EN, Wischmeyer PE. Lactobacillus rhamnosus GG improves outcome in experimental Pseudomonas aeruginosa pneumonia: potential role of regulatory T cells. Shock. 2013;40(6):496-503.

12. Bo L, Li J, Tao T, Bai Y, Ye X, Hotchkiss RS, et al. Probiotics for preventing ventilator-associated pneumonia. Cochrane Database Syst Rev. 2014;10: CD009066.

13. Johnstone J, Heels-Ansdell D, Thabane L, Meade M, Marshall J, Lauzier F, et al. The Canadian Critical Care Trials G. Evaluating probiotics for the prevention of ventilator-associated pneumonia: a randomised placebocontrolled multicentre trial protocol and statistical analysis plan for PROSPECT. BMJ Open. 2019:9(6):e025228.

14. Izumo T, Maekawa T, Ida M, Noguchi A, Kitagawa Y, Shibata H, et al. Effect of intranasal administration of Lactobacillus pentosus S-PT84 on influenza virus infection in mice. Int Immunopharmacol. 2010;10(9):1101-6.

15. Dyer KD, Drummond RA, Rice TA, Percopo CM, Brenner TA, Barisas DA, et al. Priming of the respiratory tract with Immunobiotic Lactobacillus plantarum limits infection of alveolar macrophages with recombinant pneumonia virus of mice (rK2-PVM). J Virol. 2016;90(2):979-91.

16. Fangous MS, Alexandre Y, Hymery N, Gouriou S, Arzur D, Blay GL, et al. Lactobacilli intra-tracheal administration protects from Pseudomonas aeruginosa pulmonary infection in mice - a proof of concept. Benefic Microbes. 2019:10(8):893-900.

17. Fangous MS, Lazzouni I, Alexandre Y, Gouriou S, Boisrame S, Vallet S, et al. Prevalence and dynamics of Lactobacillus $s p$. in the lower respiratory tract of patients with cystic fibrosis. Res Microbiol. 2018;169(4-5):222-6.

18. Racedo S, Villena J, Medina M, Aguero G, Rodriguez V, Alvarez S. Lactobacillus casei administration reduces lung injuries in a Streptococcus pneumoniae infection in mice. Microbes Infect. 2006;8(9-10):2359-66.

19. Belkacem N, Bourdet-Sicard R, Taha MK. Lactobacillus paracasei feeding improves the control of secondary experimental meningococcal infection in flu-infected mice. BMC Infect Dis. 2018;18(1):167.

20. Tanaka A, Seki M, Yamahira S, Noguchi H, Kosai K, Toba M, et al. Lactobacillus pentosus strain b240 suppresses pneumonia induced by Streptococcus pneumoniae in mice. Lett Appl Microbiol. 2011;53(1):35-43. 
21. Kechaou N, Chain F, Gratadoux JJ, Blugeon S, Bertho N, Chevalier C, et al. Identification of one novel candidate probiotic Lactobacillus plantarum strain active against influenza virus infection in mice by a large-scale screening. Appl Environ Microbiol. 2013;79(5):1491-9.

22. Khailova L, Petrie B, Baird CH, Dominguez Rieg JA, Wischmeyer PE. Lactobacillus rhamnosus GG and Bifidobacterium longum attenuate lung injury and inflammatory response in experimental sepsis. PLoS One. 2014; 9(5):e97861.

23. Cangemi de Gutierrez R, Santos V, Nader-Macias ME. Protective effect of intranasally inoculated Lactobacillus fermentum against Streptococcus pneumoniae challenge on the mouse respiratory tract. FEMS Immunol Med Microbiol. 2001;31(3):187-95.

24. Cangemi de Gutierrez RC, Santos de Araoz VS, Nader-Macias ME. Effect of intranasal administration of Lactobacillus fermentum on the respiratory tract of mice. Biol Pharm Bull. 2000;23(8):973-8.

25. Gabryszewski SJ, Bachar O, Dyer KD, Percopo CM, Killoran KE, Domachowske $J B$, et al. Lactobacillus-mediated priming of the respiratory mucosa protects against lethal pneumovirus infection. J Immunol. 2011;186(2):1151-61.

26. Garcia-Crespo KE, Chan CC, Gabryszewski SJ, Percopo CM, Rigaux P, Dyer $K D$, et al. Lactobacillus priming of the respiratory tract: heterologous immunity and protection against lethal pneumovirus infection. Antivir Res. 2013;97(3):270-9.

27. Budden KF, Gellatly SL, Wood DL, Cooper MA, Morrison M, Hugenholtz P, et al. Emerging pathogenic links between microbiota and the gut-lung axis. Nat Rev Microbiol. 2017;15(1):55-63.

28. Mohn KG, Smith I, Sjursen H, Cox RJ. Immune responses after live attenuated influenza vaccination. Hum Vaccin Immunother. 2018;14(3):571-8.

29. Laino J, Villena J, Suvorov A, Zelaya H, Ortiz Moyano R, Salva S, et al. Nasal immunization with recombinant chimeric pneumococcal protein and cell wall from immunobiotic bacteria improve resistance of infant mice to Streptococcus pneumoniae infection. PLoS One. 2018;13(11):e0206661.

30. Cangemi de Gutierrez R, Santos VM, Nader-Macias ME. Colonization capability of lactobacilli and pathogens in the respiratory tract of mice: microbiological, cytological, structural, and ultrastructural studies. Methods Mol Biol. 2004;268:373-85.

31. Santos V, Cangemi R, Winik B, Nader ME. Ultrastructural studies on the respiratory tract of mice inoculated intranasally with $L$. fermentum. Biocell. 2001;25(2):121-9.

32. Koh AY, Priebe GP, Ray C, Van Rooijen N, Pier GB. Inescapable need for neutrophils as mediators of cellular innate immunity to acute Pseudomonas aeruginosa pneumonia. Infect Immun. 2009;77(12):5300-10.

33. Sadikot RT, Zeng H, Joo M, Everhart MB, Sherrill TP, Li B, et al. Targeted immunomodulation of the NF-kappaB pathway in airway epithelium impacts host defense against Pseudomonas aeruginosa. J Immunol. 2006; 176(8):4923-30.

34. Sawa T, Corry DB, Gropper MA, Ohara M, Kurahashi K, Wiener-Kronish JP. IL10 improves lung injury and survival in Pseudomonas aeruginosa pneumonia. J Immunol. 1997;159(6):2858-66.

35. Vargas Garcia CE, Petrova M, Claes IJ, De Boeck I, Verhoeven TL, Dilissen E, et al. Piliation of Lactobacillus rhamnosus GG promotes adhesion, phagocytosis, and cytokine modulation in macrophages. Appl Environ Microbiol. 2015;81(6):2050-62.

36. Rocha-Ramirez LM, Perez-Solano RA, Castanon-Alonso SL, Moreno Guerrero SS, Ramirez Pacheco A, Garcia Garibay M, et al. Probiotic Lactobacillus strains stimulate the inflammatory response and activate human macrophages. J Immunol Res. 2017;2017:4607491.

37. Doron S, Snydman DR. Risk and safety of probiotics. Clin Infect Dis. 2015; 60(Suppl 2):S129-34

38. Cotran RS, Kumar V, Collins T. Robbins pathologic basis of disease. 6th ed. Philadelphia, PA: W.B. Saunders Company; 1999.

39. Dickson RP, Erb-Downward JR, Freeman CM, McCloskey L, Falkowski NR, Huffnagle GB, et al. Bacterial Topography of the Healthy Human Lower Respiratory Tract. mBio. 2017;8(1):e02287-16.

40. Dickson RP, Erb-Downward JR, Martinez FJ, Huffnagle GB. The microbiome and the respiratory tract. Annu Rev Physiol. 2016;78:481-504.

41. Morris A, Beck JM, Schloss PD, Campbell TB, Crothers K, Curtis JL, et al. Comparison of the respiratory microbiome in healthy nonsmokers and smokers. Am J Respir Crit Care Med. 2013;187(10):1067-75.

42. Rosas-Salazar C, Shilts MH, Tovchigrechko A, Schobel S, Chappell JD, Larkin EK, et al. Nasopharyngeal Lactobacillus is associated with a reduced risk of childhood wheezing illnesses following acute respiratory syncytial virus infection in infancy. J Allergy Clin Immunol. 2018;142(5):1447-56 e9.

43. Sze MA, Dimitriu PA, Hayashi S, Elliott WM, McDonough JE, Gosselink JV, et al. The lung tissue microbiome in chronic obstructive pulmonary disease. Am J Respir Crit Care Med. 2012;185(10):1073-80.

44. Mourits VP, Wijkmans JC, Joosten LA, Netea MG. Trained immunity as a novel therapeutic strategy. Curr Opin Pharmacol. 2018;41:52-8.

45. Maassen CB, van Holten-Neelen C, Balk F, den Bak-Glashouwer MJ, Leer RJ, Laman JD, et al. Strain-dependent induction of cytokine profiles in the gut by orally administered Lactobacillus strains. Vaccine. 2000;18(23):2613-23.

46. van den Broek MFL, De Boeck I, Claes IJJ, Nizet V, Lebeer S. Multifactoria inhibition of lactobacilli against the respiratory tract pathogen Moraxella catarrhalis. Benefic Microbes. 2018;9(3):429-39.

47. Lagrafeuille R, Miquel S, Balestrino D, Vareille-Delarbre M, Chain F, Langella $P$, et al. Opposing effect of Lactobacillus on in vitro Klebsiella pneumoniae in biofilm and in an in vivo intestinal colonisation model. Benefic Microbes. 2018;9(1):87-100.

48. Le Berre R, Nguyen S, Nowak E, Kipnis E, Pierre M, Quenee L, et al. Relative contribution of three main virulence factors in Pseudomonas aeruginosa pneumonia. Crit Care Med. 2011;39(9):2113-20.

49. Kipnis E, Sawa T, Wiener-Kronish J. Targeting mechanisms of Pseudomonas aeruginosa pathogenesis. Med Mal Infect. 2006;36(2):78-91.

50. Holloway BW, Krishnapillai V, Morgan AF. Chromosomal genetics of Pseudomonas. Microbiol Rev. 1979;43(1):73-102.

51. Schaber JA, Carty NL, McDonald NA, Graham ED, Cheluvappa R, Griswold $J A$, et al. Analysis of quorum sensing-deficient clinical isolates of Pseudomonas aeruginosa. J Med Microbiol. 2004;53(Pt 9):841-53.

52. Huet O, Ramsey D, Miljavec S, Jenney A, Aubron C, Aprico A, et al. Ensuring animal welfare while meeting scientific aims using a murine pneumonia model of septic shock. Shock. 2013;39(6):488-94.

\section{Publisher's Note}

Springer Nature remains neutral with regard to jurisdictional claims in published maps and institutional affiliations.

Ready to submit your research? Choose BMC and benefit from:

- fast, convenient online submission

- thorough peer review by experienced researchers in your field

- rapid publication on acceptance

- support for research data, including large and complex data types

- gold Open Access which fosters wider collaboration and increased citations

- maximum visibility for your research: over $100 \mathrm{M}$ website views per year

At BMC, research is always in progress.

Learn more biomedcentral.com/submissions 